\title{
An Update on ISoP Special Interest Groups (SIGs)
}

\author{
Deirdre McCarthy ${ }^{1} \cdot$ Priya Bahri $^{2} \cdot$ Joanne Barnes $^{3} \cdot$ Jean-Christophe Delumeau $^{4}$. \\ Brian Edwards $^{5} \cdot$ Mira Harrison-Woolrych ${ }^{6}$
}

Published online: 4 October 2017

(c) Springer International Publishing AG 2017

\section{Introduction}

Special interest groups or 'SIGs' are becoming an increasingly important part of the International Society of Pharmacovigilance (ISoP). SIGs are groups of ISoP members working on a specific pharmacovigilance topic relevant to the scope and aims of ISoP, which are primarily education, training and promotion of research for the benefit of patients. Currently, there are SIGs on women's medicines, risk communication, and risk minimisation for Asian countries. A new SIG on herbal and traditional medicines was created in April 2017 and will be launched formally at the ISoP 2017 annual conference in Liverpool, UK. In this article, we describe the ongoing work of ISoP

The views expressed in this article represent those of the authors, who contribute to these ISoP activities in their personal capacity, and do not necessarily represent the views or practices of the authors' employers or any other party.

Deirdre McCarthy

deemccarthy1234@gmail.com

1 Lifecycle Safety, QuintilesIMS, Cambridge, MA, USA

2 Pharmacovigilance and Epidemiology Department, European Medicines Agency (EMA), London, UK

3 School of Pharmacy, Faculty of Medical and Health Sciences, University of Auckland, Auckland, New Zealand

4 Head of Pharmacovigilance Policy Strategy (Global), Bayer (South East Asia) Pte Ltd., OCBC Centre East, 63 Chulia Street, Singapore, Singapore

5 NDA Regulatory Science Ltd, Leatherhead, UK

6 Dunedin School of Medicine, University of Otago, Dunedin, New Zealand with the existing SIGs and introduce the two new SIGs under development.

SIGs operate as global networks without regional limitations. While they exist primarily to connect ISoP members, they may also engage non-ISoP members with special expertise in the topic concerned. In addition, a SIG may work together on its specific topic with other groups, associations or societies outside ISoP, after agreement by the ISoP Executive Committee (EC). The instigator or instigators of a SIG usually take the lead to get the group off the ground and invite interested parties to become founding members and begin preliminary work. A coordinator or lead person is then chosen who cooperates closely with the ISoP Education and Training Program team and the ISoP secretariat. The coordinators of the current and developing SIGs are summarised in Table 1.

As with all other ISoP activities, work within and for each SIG is voluntary. ISoP is a not-for-profit organisation with no paid positions other than the ISoP executive secretary, and ISoP members, including EC and board members, donate their time to this work. This year, the ISoP EC has strengthened its support of the SIG community by creating an over-arching SIG coordinator role and appointing a member of the advisory board to this position. This role is described in Sect. 4.

\section{How Special Interest Groups (SIGs) are Created}

A SIG can be created by at least five ISoP members who decide to engage and work together on a specific topic that is within the scope and aims of ISoP. These members have to submit to the ISoP EC a proposal for establishing the new SIG. The EC then considers whether the proposed activities are consistent with the aims of ISoP, will 
Table 1 Active and in-progress special interest groups

\begin{tabular}{lll}
\hline ISoP SIGs & Coordinators & Status (August 2017) \\
\hline Women's medicines & Mira Harrison-Woolrych & Active \\
Risk communication & Priya Bahri & Active \\
Risk minimisation methods for Asian countries & Jean-Christophe Delumeau & Active \\
Herbal and traditional medicines & Joanne Barnes & Active \\
Pharmacovigilance professional qualification framework & Jan Petracek & Underway \\
Medication errors & Brian Edwards and Ian Wong & Underway \\
\hline
\end{tabular}

$I S o P$ International Society of Pharmacovigilance, $S I G$ special interest group

strengthen ISoP as a whole, and will provide benefit to patients. If so, the SIG will be formally approved and it can begin working towards an official launch.

\section{Activities}

ISoP SIGs enable members to be personally involved in discussing key issues in their area of interest and to network with others within ISoP. SIGs provide an opportunity to progress one's own professional area of interest and be involved in publications, presentations and research opportunities with the overarching goal of promoting patient safety. This may all lead to the development of papers, opinions and statements that may become official and public ISoP documents. Such documents need to be adopted by the EC prior to release or publication. SIGs may also support conferences, symposia, training courses or workshops. SIGs can present their activities at annual meetings or other ISoP events. SIG members can also combine their expertise in publishing papers in scientific journals for a wider audience on behalf of the respective SIG. Examples of specific activities undertaken by the current SIGs are given in Sect. 6.

Members of SIGs are encouraged to collaborate in the recruitment of new members to help achieve a 'critical mass' and make SIGs viable. The contribution of external experts who may support the activities and goals of the SIG is also welcomed and, in this regard, the ISoP SIGs work closely with international experts in many areas of pharmacovigilance. SIGs retain an overall commitment to act as important sources of the information that helps achieve ISoP's mission.

\section{The ISoP SIG Coordinator}

As mentioned in Sect. 1, an overall SIG coordinator is in place to support SIG set-up and activities. This role-undertaken by Deirdre McCarthy during the 2016 to 2019 term-is appointed by the EC from the members of the ISoP board every time a new EC is elected. Key aspects of this role are as follows:

- Exploring topic areas that may benefit from a SIG.

- Reviewing and evaluating new SIG proposals received from ISoP members, and submitting to the ISoP board.

- Supporting the set-up of any new SIGs that are approved by the ISoP board until they are formally launched and running smoothly.

- Providing support and encouragement in writing articles in the official journal of the society, Drug Safety.

The ISoP SIG coordinator ensures through regular communication with each SIG leadership team that each SIG is supported and is active. The coordinator also provides regular reports to the EC, ensures the SIG section of the ISoP website is updated and helps to promote the SIGs wherever possible.

\section{Contribution of SIGs to the Annual Meeting}

SIGs contribute to the annual meeting in a number of ways. Discussion on a particular SIG topic at the meeting can generate interest and discussion from new ISoP members and other experts and support the continued growth of the SIG. Contributions may take the form of a pre-conference training course, a stand-alone podium presentation or an entire session. At ISoP 2017 in Liverpool, a session for all ISoP SIGs will be held during the plenary program, and the Women's Medicines SIG will also chair a specific session (http:// isop2017liverpool.org/).

The ISoP annual meeting is also a great opportunity to launch new SIGs. At the Liverpool meeting, three new SIGs will be formally launched: the new Herbal and Traditional Medicines SIG, the Pharmacovigilance Professional Qualifications SIG, and the Medication Errors SIG (for more information on all three, see Sect. 6). ISoP members are warmly invited to attend this exciting event. 
This year's ISoP meeting also includes a plenary lecture, 'Pharmacovigilance for herbal and traditional medicines: towards solutions and innovations', reflecting the importance of and interest in this topic.

\section{Overview of Current Active SIGs}

The ISoP SIGs are summarised in Table 1. In this section, we review those that are already active.

\subsection{Women's Medicines}

The SIG on medicines for women's health was established in 2011 by Mira Harrison-Woolrych. The members of this ISoP SIG have international expertise and interest in these products and the populations in which they may be used, for example, medicines taken during pregnancy.

The key philosophy of this SIG is that the safe use of medicines by women for their reproductive and other health and wellbeing improves not only women's lives but also the health and well-being of their families, including babies, children and men, and hence makes an important contribution to communities and societies.

The women's medicines SIG welcomes all ISoP members with an interest in this area, regardless of their gender, race, or place of work. The aims of the SIG are as follows:

- To share relevant new publications_-including research findings, regulatory actions, news items and other information on women's medicines issues-that have been identified worldwide.

- To discuss and debate key issues arising relating to the safe use of women's medicines, within both the SIG and wider audiences.

- To keep women's medicines at the heart of ISoP's core values to improve the effective and safe use of medicines for all people in all countries of the world.

- To improve the quality of information available regarding women's medicines (including use during pregnancy) in published material where benefits and risks are communicated, including patient information leaflets for licensed medicines/devices and information provided to research participants in clinical trials.

- To promote access to effective and safe contraceptive products worldwide, with objective and accurate information about the benefits and risks of use.

- To promote development of pregnancy registers for medicines, especially those taken by women of reproductive age and including drugs used for in vitro fertilisation (IVF) treatments.
- To work with other groups both within ISoP (e.g. the CommSIG) and outside ISoP (e.g. professional groups, patient organisations, World Health Organisation International Society of Epidemiology) that are also concerned about the safe use of women's medicines.

- To communicate the work of the SIG as widely as possible, including at ISoP meetings and other professional conferences and in journal articles and other publications.

Since 2011, there have been lively sessions on medicines for women's health at ISoP annual meetings around the world.

In 2015, members of this ISoP SIG were authors and editor of the book Medicines for Women published by Springer International [1]. This is the first textbook to focus on this area of prescribing and pharmacovigilance and includes 19 chapters covering specific groups of products (e.g. contraceptive devices, bisphosphonates), specific subpopulations (e.g. adolescent women, pregnant women) and broader concerns, such as prescribing for women in general practice and communication of benefits and risks of women's medicines.

\subsection{Risk Communication}

The Risk Communication SIG (known as the CommSIG) was founded by Priya Bahri and others and began its activities in 2014. The group was set up because communication is vital for pharmacovigilance. No pharmacovigilance goals, nor the wider vision of patient safety, can be achieved without enhancing communication with patients, healthcare providers and other influential parties at the expert level. As a result, ISoP has included communication topics in the programme of each of its annual meetings since 2006, which led to some ISoP members developing a themed edition of Drug Safety on risk communication in 2012 [2]. This edition discussed proposals for improving communication practices from worldwide experience [3]. The CommSIG committed to build on this work and, as a starting point, consolidated previous ISoP discussions and proposals, further defining its main objectives and future paths in a publication in 2015. It has invited ISoP members to contribute and support the CommSIG in connecting with experts outside ISoP [4].

The CommSIG's mandate has the following scope, which has never previously been attempted:

- To establish risk communication as a sub-discipline of safety surveillance and risk management of medicines; provide for multi-disciplinary exchange, learning and practice development; and promote related research.

- To outreach to others, such as patient groups, healthcare providers, drug information providers and the 
media, and connect with experts from the communications, social and healthcare sciences.

- Overall, to support the development of improved risk communication practices in various pharmacovigilance settings worldwide with a view to facilitating informed therapeutic choices, safe use of medicines and the credibility of the regulatory system.

To live up to these goals, the CommSIG organised a plenary session "Patient safety through dialogue: how to better connect pharmacovigilance and healthcare?", which included two external experts, at the ISoP annual meeting in 2015 [5]. A training paper summarising the contributions in relation to improving verbal and numerical risk expression is due for publication. In 2016, the CommSIG provided the chair to a session "Risk communication 'Snakes and Ladders' and a strategic approach to risk minimisation in healthcare." Speakers from India, Thailand, Singapore and Italy presented their local efforts and successes, and an article summarising the experiences and motivating others for similar engagement is currently under preparation. The CommSIG has also contributed to ISoP's training activities and has taken the initiative to develop training video clips in 2017-2018.

The CommSIG has a standing interaction with the International Society for Pharmacoepidemiology (ISPE) and connects with experts from the social and communications sciences, those active in patient safety and human factors, and with representatives from patient, healthcare provider and media organisations. The CommSIG is also dedicated to creating synergies with other ISoP SIGs and chapters and other international organisations and learned societies.

\subsection{Risk Minimisation Methods for Asian Countries}

In existence since 2015, the Risk Minimisation Methods for Asian Countries SIG is a global group with a focus on the very diverse and swiftly developing Asian region. It is coordinated by Jean-Christophe Delumeau, an ISoP EC member based in Singapore. This SIG also has a chairperson (Herve le Louet) and a methodologist (Yola Moride).

The mandates of this SIG are as follows:

- To provide a forum to exchange experience on risk minimisation methods, tools and operational practices with a focus on Asian countries.

- To elaborate an array of solutions for risk-based risk minimisation interventions (RMIs) that can be operated in an array of counties with diverse healthcare systems.

- To engage with the development of affordable solutions, using mobile devices, capable of raising awareness on the adequate use of medicines and/or support RMIs.

- To develop training materials to promote the deployment of those solutions elaborated by the group.

- To communicate about those solutions and advocate for their deployment in the different sub-regions of Asia and beyond.

After analysing the array of risk minimisation practices applied in different Asian countries, and developing a comprehensive model of a post-approval vigilance program (PAVP), the SIG engaged with a Delphi study programme conducted by Yola Moride. This study aims to determine the circumstances that should lead to consideration of implementing an RMI and determine their degree of stringency in consideration of the characteristics and capability of the healthcare system.

To overcome the lack of literature available on such criteria, a preliminary interview phase proved to be necessary to collect the practice of experienced experts involved in risk management planning activities. The preDelphi interview phase is now completed, and the core component of the Delphi study can start, involving a minimum of 25 responding panellists from a wide range of Asian countries with diverse healthcare systems.

In parallel, members of the SIG are developing, as a satellite project, the Aspidium App: a versatile application for smartphones intended for patients and healthcare professionals. This app is aimed to enhance the impact of routine risk minimisation or support educational RMIs for specific products, including generics. This app is capable of hosting contents for multiple products. The supporting platform is designed to be customised and deployed on a per-country basis. A poster on this topic will be presented at the 17th annual ISoP meeting in Liverpool on 16 October 2017.

\subsection{Herbal and Traditional Medicines}

The Herbal and Traditional Medicines SIG was founded in 2017. Its overall aims are to provide a focal point for ISoP members interested in these types of preparations to share and provide information on relevant issues and developments and to support and promote progress in pharmacovigilance for these and other natural health products.

The key objectives are as follows:

- To discuss issues in herbal and traditional medicines' safety and pharmacovigilance and work collaboratively towards finding solutions.

- To raise awareness of issues in herbal and traditional medicines' safety and pharmacovigilance among relevant organisations, including those representing patient 
groups, healthcare providers, medicines information providers, media and others.

- To serve as a conduit for ISoP members wishing to initiate or be involved with collaborative research or other projects of relevance to the herbal and traditional medicines area.

Experts in this area believe action is needed on numerous issues relating to herbal and traditional medicines. These preparations and products are a popular healthcare choice in many countries and, in some parts of the world, herbal and traditional medicines are the only or main form of health treatment available to many people. However, comprehensive information on the safety (and efficacy) of many natural health products is lacking. Furthermore, existing pharmacovigilance systems have limitations with respect to collecting reports of suspected adverse drug reports associated with the use of these products, including that they receive few reports from patients and practitioners using herbal and traditional medicines.

Part of the work of the Herbal and Traditional Medicines SIG will be to identify and collate the issues, develop an 'issues' agenda, and determine what action is needed. For example, there are difficulties in coding and classifying herbal and traditional medicines and other natural health products and the potential to improve and standardise how this is done internationally. Another area of interest is around stimulating patient reporting of suspected adverse reactions associated with herbal and traditional medicines and other natural health products. Members of the SIG are also invited to raise and discuss other issues relating to herbal and traditional medicines they feel are of importance.

As with other SIGs, where other international organisations and learned societies are working on relevant issues, the ISoP Herbal and Traditional Medicines SIG will aim to liaise and work collaboratively. Likewise, the SIG is committed to creating productive relationships with other ISoP SIGs and chapters.

The group aims to share interesting news items, research findings, regulatory actions and other information on herbal medicines issues that have been identified worldwide. The purpose of this activity is to share information, stimulate debate, and possibly lead to the development of new projects.

\section{Current Developments}

During 2017, the groundwork has been laid for two additional SIGs. The first proposed new SIG is to explore issues relating to professional qualifications in pharmacovigilance
(Pharmacovigilance Professional Qualification Framework SIG), to be headed by ISoP board member Jan Petracek. ISoP is significantly involved in the training of pharmacovigilance professionals worldwide, and we believe this is an additional important topic in which ISoP can play a key global role. There is a need for harmonisation in professional pharmacovigilance certifications, especially in the area of better defining pharmacovigilance professional career structures across the many pharmacovigilance career paths.

Many professions have structures that allow for further specialisation, recognition and development. The profession of pharmacovigilance does not currently have a structure that is globally recognized and accepted. This can potentially create inconsistent performance in some parts of the pharmacovigilance world, challenges with decision making and variable recognition of the pharmacovigilance profession, amongst other issues.

This new SIG aims to build on the successes of the existing ISoP pharmacovigilance curriculum [6], certification programmes and academic degrees and hopes to create an internationally recognised qualification framework for all career tracks in pharmacovigilance.

In ISoP, we believe that pharmacovigilance as a profession has now reached a maturity that is suitable for creation of the framework.

The SIG will be officially launched during the 17th ISoP annual meeting in Liverpool.

The second new SIG will cover medication errors and will be co-led by Brian Edwards and Ian Wong, both from the UK. The mission of this new SIG in development is to provide an opportunity for ISoP members and like-minded collaborators to network globally in a professional and neutral environment to share evidence and solutions to tackle medication errors, thereby continuing systematic improvement in optimising the use of medicines in the interests of healthcare professionals, patients and their families. Close liaison with the World Health Organization Medication Safety initiative (http://www.who.int/ patientsafety/medication-safety/en/) will be pursued. The following goals and objectives of the SIG are still under discussion:

- Create opportunities for those researching and investigating medication errors to network in a friendly and mutually supportive environment and publish their research using good-quality outlets.

- Support healthcare professionals with scientific evaluation of medication errors and how to prevent them.

- Help regulatory authorities with medication errors guidance evaluation.

- Organise training and tutorials to provide effective support to pharmacovigilance centres to extend their 
role in detecting and preventing medication errors through individual case safety reports (ICSRs) by using specific methods and tools.

- Provide a neutral and impartial forum for all stakeholders, including patients, to ask difficult questions and obtain answers within a reasonable timeline.

- Through social media and other forms of communication, build on current activities to provide a platform for discussion and generation of new research and ideas.

- Explore the need for meetings, education and affordable training, such as encouraging abstracts for the annual meeting and contributing to ISoP training meetings.

\section{Advantages of Joining a SIG}

Membership of a SIG has numerous advantages, such as advancing research interests, contributing to publications, developing and promoting scientific standards, becoming part of a professional community and expanding one's network in areas of interest.

The ISoP SIGs are here to progress the safe use of medicines for the benefit of patients. Anyone wishing to join one of the SIGs should feel free to contact the respective SIG leader at the ISoP contact email (administration@isoponline.org). Further information is also available on the ISoP website (http://isoponline.org/specialinterest-groups/).

Acknowledgements With thanks to Sophie Spence, Executive Secretary of ISoP for her ongoing support and assistance with all ISoP SIGs, to Ulrich Hagemann of Germany for creation of the original SIG guidelines and to Jan Petracek of the Czech Republic for developing the Pharmacovigilance Professional Qualification Framework SIG.

\section{Compliance with Ethical Standards}

Funding No funding has been received for activities related to the development of this article.

Conflicts of interest Deirdre McCarthy, Priya Bahri, Jean-Christophe Delumeau and Mira Harrison-Woolrych have no conflicts of interest that are directly relevant to the content of this article. Joanne Barnes receives royalties from Elsevier in relation to a co-authored text Fundamentals of Pharmacology and Phytotherapy. She also sits on the expert advisory subcommittee on the Permitted Substances List (Natural Health and Supplementary Products Regulations) to the New Zealand Ministry of Health. She is an honorary consultant and herbal safety signal reviewer, World Health Organisation, Uppsala Monitoring Centre. She is an associate editorial board member and international board member of the following journals: Phytochemistry Letters, Phytotherapy Research, Drug Safety, Drug Safety - Case Reports, International Journal of Pharmacy Practice and Complementary Therapies in Medicine. She is a member of the Medicinal Plant Names Services user group, Royal Botanic Gardens, Kew, London, UK, and a member of the Advisory Board of the American Botanical Council. Brian Edwards is a pharmaceutical consultant with NDA Regulatory Science Ltd.

\section{References}

1. Harrison-Woolrych M, editor. Medicines for women. 1st ed. New York: Springer Ltd; 2015.

2. Drug Safety. 2012;35(11). https://link.springer.com/journal/40264/ 35/11/page/1. Last accessed 11 Sept 2017.

3. Bahri P, Harrison-Woolrych M. Focusing on risk communication about medicines: why now? [editorial]. Drug Saf. 2012;35:971-5.

4. Bahri P, Dodoo AN, Edwards BD, Edwards IR, Fermont I, Hagemann U, Hartigan-Go K, Hugman B, Mol PG, On behalf of the ISoP CommSIG. The ISoP CommSIG for improving medicinal product risk communication: a new special interest group of the International Society of Pharmacovigilance. Drug Saf. 2015;38:621-7.

5. Bahri P, Edwards B. Patient safety through dialogue: how to better connect pharmacovigilance and healthcare? [abstract]. Drug Saf. 2015;38:959.

6. Beckmann J, Hagemann U, Bahri P, Bate A, Boyd IW, Dal Pan GJ, et al. Teaching pharmacovigilance: the WHO-ISoP core elements of a comprehensive modular curriculum. Drug Saf. 2014;37:743-59. 